\title{
Ultrasound combined with targeted cationic microbubble-mediated angiogenesis gene transfection improves ischemic heart function
}

\author{
QING ZHOU, QING DENG, BO HU, YI-JIA WANG, JIN-LING CHEN, \\ JING-JING CUI, SHENG CAO and HONG-NING SONG
}

Department of Ultrasound Imaging, Renmin Hospital, Wuhan University, Wuhan, Hubei 430060, P.R. China

Received October 21, 2015; Accepted December 19, 2016

DOI: $10.3892 /$ etm.2017.4270

\begin{abstract}
The present study aimed to construct targeted cationic microbubbles (TCMBs) by synthesizing cationic microbubbles conjugated to an intercellular adhesion molecule-1 (ICAM-1) antibody, and then to use the TCMBs to deliver the angiopoietin-1 (Ang-1) gene into infarcted heart tissue using ultrasound-mediated microbubble destruction. It was hypothesized that the TCMBs would accumulate in higher numbers than non-targeted cationic microbubbles (CMBs) in the infarcted heart, and would therefore increase the efficiency of targeted Ang-1 gene transfection and promote angiogenesis. The results of the study demonstrated that the ability of TCMBs to target inflammatory endothelial cells was 18.4-fold higher than that of the CMBs in vitro. The accumulation of TCMBs was greater than that of CMBs in TNF- $\alpha$-stimulated human umbilical cord veins, indicated by a $212 \%$ higher acoustic intensity. In vivo, the TCMBs specifically accumulated in the myocardial infarct area in a rabbit model. Three days after ultrasound microbubble-mediated gene transfection, Ang-1 protein expression in the TCMB group was 2.7-fold higher than that of the CMB group. Angiogenesis, the thickness of the infarct region and the heart function of the TCMB group were all significantly improved compared with those in the CMB and control groups at 4 weeks following gene transfection (all $\mathrm{P}<0.01)$. Therefore, the results of the current study demonstrate that ultrasound-mediated TCMB destruction effectively delivered the Ang-1 gene to the infarcted myocardium, resulting in improved cardiac morphology and function in the animal model. Ultrasound-mediated TCMB destruction is a promising strategy for improving gene therapy in the future.
\end{abstract}

Correspondence to: Dr Qing Zhou, Department of Ultrasound Imaging, Renmin Hospital, Wuhan University, 238 Jiefang Road, Wuhan, Hubei 430060, P.R. China

E-mail: qingzhou128@163.com

Key words: ultrasound, targeted microbubble, intercellular adhesion molecule-1, gene transfection, angiogenesis, myocardial infarction

\section{Introduction}

Clinical therapeutic revascularization techniques have improved considerably in recent years; however, treatment of extensive myocardial infarction and refractory myocardial ischemia remains relatively difficult due to poor long-term outcomes (1). It has been demonstrated in a number of experimental and pre-clinical studies that gene-based therapeutic angiogenesis is feasible $(2,3)$. However, the low efficiency of gene delivery to the target tissue limits the potential of this type of therapy $(4,5)$.

Ultrasound-targeted microbubble destruction (UTMD) has garnered increased attention as a potential method for gene delivery in recent years. In an ultrasound field, microbubbles undergo either stable cavitation or inertial cavitation, which results in oscillatory shear, high-pressure microstream and physical stretching of endothelial cells. These bioeffects can cause sonoporation on the surface membranes of cells, increase blood vessel wall permeability and facilitate gene uptake (6-8). Studies investigating the UTMD-mediated delivery of angiogenesis genes to treat ischemic heart disease have attracted considerable attention (9-11). However, this novel gene transfection technique requires further development prior to its application as a clinical treatment $(12,13)$. Gene concentration at the site of sonoporation may cause unsatisfactory therapeutic effects. Although focused ultrasound destruction of microbubbles can, to a certain extent, provide targeted gene release, microbubbles that lack a tissue-specific ligand linkage will distribute throughout the body via the blood circulation. Consequently, the amount of the therapeutic gene reaching the target tissue is limited, resulting in poor gene uptake $(12,13)$. One possible method of increasing the therapeutic potential of UTMD is to specifically target microbubbles to the tissue of interest by conjugating receptor ligands or antibodies to their surface (14).

Intercellular adhesion molecule-1 (ICAM-1), which is expressed by inflammatory endothelial cells, participates in leukocyte rolling and adhesion (15). Overexpression of ICAM-1 is considered to be a marker of endothelial cell dysfunction or injury and has been observed in a number of related diseases, including thrombosis and myocardial infarction $(15,16)$. The present study attempted to conjugate an ICAM-1 antibody to 
cationic microbubbles to facilitate attachment to the injured vasculature, as well as boosting the regional microbubble population around the injured vascular endothelial cells in the infarcted myocardium. It was hypothesized that the use of an ICAM-1 antibody would allow greater numbers of microbubbles to accumulate in the injured vascular endothelial tissue and promote gene transfer through the endothelial gap, resulting in increased gene uptake and more efficient intravenous gene transfection.

Angiopoietin 1 (Ang-1) serves an important role in angiogenesis and its effects following myocardial infarction last longer than those of vascular endothelial growth factor (17,18). In addition, Ang-1 may reduce the vascular permeability caused by endothelial growth factors, promote vessel maturation and ultimately improve myocardial perfusion $(19,20)$. Therefore, in the present study, Ang-1 was selected as the therapeutic gene to be tested in a model of myocardial infarction and targeted cationic microbubbles (TCMBs) loaded with an ICAM-1 monoclonal antibody were used as the carriers of the Ang-1 gene. It was hypothesized that TCMBs would enhance gene delivery to the ischemic area, thus increasing therapeutic angiogenesis and improving infarcted heart function.

\section{Materials and methods}

Preparation of the TCMBs. The cationic microbubbles were composed of a perfluoropropane gas core encapsulated by 1,2-distearoyl-sn-glycero-3-phosphocholine (DSPC; Sigma-Aldrich; Merck Millipore, Darmstadt, Germany), 1,2-distearoyl-sn-glycero-3-phosphoethanolamine-N-[maleimide (polyethylene glycol)-2000] (DSPE-PEG2000; Avanti Polar Lipids, Alabaster, AL, USA), 1,2-dipalmitoyl-sn-glycero-3-phosphate (DPPA; Sanofi

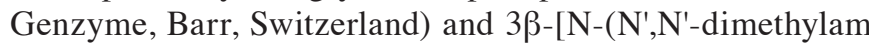
inoethane)-carbamoyl] cholesterol hydrochloride (DC-Chol; Avanti Polar Lipids) [molar ratio (39:1:40:20\%)]. DC-Chol provided a positively charged surface for the cationic microbubbles. For the biotinylated microbubbles, DSPE-PEG2000 biotin (Avanti Polar Lipids, Inc.) was added, enabling conjugation of the targeting ligands using biotin-streptavidin binding chemistry. Following the generation of the biotinylated microbubbles, streptavidin $\left(30 \mu \mathrm{g} / 10^{8}\right.$ microbubbles; Sigma-Aldrich; Merck Millipore) was added for $30 \mathrm{~min}$ at $4^{\circ} \mathrm{C}$, as previously described (21). The biotinylated monoclonal ICAM-1 antibody (bs-4617R; 1:100; Beijing Biosynthesis Biotechnology Co., Ltd., Beijing, China) was mixed with the cationic microbubbles in a 1:100 volume ratio for $30 \mathrm{~min}$ at $4^{\circ} \mathrm{C}$. Static flotation was used to remove the unbound antibody from the microbubbles by washing three times with phosphate-buffered saline (PBS).

To confirm the conjugation of the ICAM-1 antibody to the cationic microbubbles, $100 \mu$ l fluorescein isothiocyanate (FITC)-labeled mouse anti-human immunoglobulin (Ig) G (ab49867; 1:100; Abcam, Cambridge, MA, USA) was added to $200 \mu 1$ microbubbles and mixed for $30 \mathrm{~min}$ at $4^{\circ} \mathrm{C}$. The mixture was separated by low-speed centrifugation at $4^{\circ} \mathrm{C}(400 \mathrm{x} g$ for $5 \mathrm{~min}$ ) and the upper layer was washed three times with PBS to remove the free FITC-conjugated IgG. The successful construction of TCMBs was confirmed by the presence of bright green fluorescence at the fringe of the microbubble surface, as shown using fluorescence microscopy (Olympus Corporation, Tokyo, Japan).

To analyze the characteristics of the TCMBs, non-targeted cationic microbubbles (CMBs) were used as a control. The morphology of the microbubbles was examined using bright-field and fluorescence microscopy (Olympus Corporation), the mean diameter of the microbubbles was determined by electrozone sensing (Multisizer ${ }^{\mathrm{TM}}$ version 3; Beckman Coulter, Inc., Brea, CA, USA) following the manufacturer's protocol and the zeta potential of the microbubbles was measured using a Zetasizer Nano S instrument (Malvern Instruments, Worcestershire, UK) according to the manufacturer's operating manual.

Conjugation of the DNA to the microbubbles. The Ang-1 gene plasmid was constructed by ligating the Ang-1 gene into the pcDNA3.1 vector with a cytomegalovirus promoter to induce Ang-1 expression. A total of $20 \mu \mathrm{g}$ Ang-1 plasmid was mixed with $200 \mu \mathrm{l}\left(\sim 1 \times 10^{8}\right) \mathrm{TCMB}$ or CMB in $1 \mathrm{ml}$ PBS. The mixture was incubated for $15 \mathrm{~min}$ at room temperature and then centrifuged at $37^{\circ} \mathrm{C}$ and $400 \times \mathrm{g}$ for $5 \mathrm{~min}$ to form two phases. The upper layer contained the microbubble-bound plasmid and the lower, clear layer contained the unbound plasmid. The subnatant was collected and its plasmid content was analyzed using UV spectrophotometry at $260 \mathrm{~nm}$ and was compared with a standard. The standard curve was created in house using UV spectrophotometry at $260 \mathrm{~nm}$ to detect the Ang-1 gene plasmid with a series of different concentration $(0.01$, $0.05,0.1,0.5,1.0,5.0,10.0$ and $20.0 \mu \mathrm{g} / \mathrm{ml})$. The gene-carrying efficiency of the microbubbles was defined as follows: (Total quantity of plasmid-quantity of plasmid in the subnatant)/total quantity of plasmid.

Targeting ability of TCMBs for inflammatory endothelial cells in vitro. Human umbilical vein endothelial cells (HUVECs) were extracted from the endothelium of human umbilical cord veins. The umbilical cords were acquired from the delivery room at Renmin Hospital (Wuhan, China) and the experimental process was approved by the Ethics Committee of Renmin Hospital. Briefly, the umbilical vein was filled with $20 \mathrm{ml}$ of $0.1 \%$ collagenase (Type II; cat. no. 17101015; Invitrogen; Thermo Fisher Scientific, Inc., Waltham, MA, USA) dissolved in PBS and incubated for $15 \mathrm{~min}$ at $37^{\circ} \mathrm{C}$. The collagenase solution was drained from the cord and collected. The cells in this solution were recovered via centrifugation at $37^{\circ} \mathrm{C}$ and $112 \times \mathrm{g}$ for $5 \mathrm{~min}$ and transferred to culture dishes. HUVECs were subsequently in endothelial cell medium (ECM) containing 10\% fetal bovine serum and $1 \%$ endothelial cell growth supplement (ScienCell Research Laboratories, Inc., Carlsbad, CA, USA). The cells were maintained for $24 \mathrm{~h}$ in $10-\mathrm{cm}$ culture dishes at $37^{\circ} \mathrm{C}$ in an atmosphere containing $5 \% \mathrm{CO}_{2}$. The HUVECs were subsequently treated with human recombinant tumor necrosis factor- $\alpha$ (TNF- $\alpha$; R $\& D$ systems, Inc., Minneapolis, MN, USA) to generate a model of inflammatory endothelial cells. A total of $2 \times 10^{6}$ HUVECs were cultured in ECM supplemented with various doses of TNF- $\alpha(0,10,20$ and $50 \mathrm{ng} / \mathrm{ml}$ ) at $37^{\circ} \mathrm{C}$ in an atmosphere containing $5 \% \mathrm{CO}_{2}$ for $4 \mathrm{~h}$. Western blotting was then used to detect the expression of ICAM-1. The adherent cells were lysed in $1 \mathrm{ml}$ of ice-cold tissue lysis buffer (1X Tris-buffered saline, $1.5 \%$ Triton X-100, 
$0.5 \%$ deoxycholic acid, $0.1 \%$ SDS, protease inhibitor cocktail and $1 \mathrm{mM}$ phenylmethanesulfonyl fluoride; all Sigma Aldrich; Merck Millipore) and centrifuged (12,000 x $\left.\mathrm{g}, 20 \mathrm{~min}, 4^{\circ} \mathrm{C}\right)$, following which the supernatants were collected. The protein concentration was determined using a bicinchoninic acid protein assay kit (P0010; Beyotime Institute of Biotechnology, Haimen, China). Protein samples (30 $\mu \mathrm{g} /$ lane) were separated by $10 \%$ SDS-PAGE, transferred onto polyvinylidene fluoride membranes and blocked with 5\% nonfat dry milk for $1 \mathrm{~h}$ at room temperature. Membranes were subsequently incubated with rabbit anti-human ICAM-1 primary antibody (1:200; bs-4617R; Beijing Biosynthesis Biotechnology Co., Ltd.) at $4^{\circ} \mathrm{C}$ overnight before being incubated with horseradish peroxidase-coupled secondary antibody (goat anti-rabbit IgG; 1:5,000; ab6721; Abcam) for $1 \mathrm{~h}$ at room temperature. The membranes were washed with $20 \mathrm{ml}$ TBST for 5 min 3 times and exposed to X-rays to detect the expression bands. Image $\mathrm{J}$ software (version 1.4; National Institutes of Health, Bethesda, MA, USA) was used to quantify the bands.

The HUVECs were cultured in an inverted parallel-flow chamber filled with extracellular matrix (Collagen I from rat tail tendons; C3867; Sigma-Aldrich; Merck Millipore) for microbubble attachment. When the HUVECs reached $70 \%$ confluence $\left(\sim 2 \times 10^{6}\right)$, they were activated with $50 \mathrm{ng} / \mathrm{ml} \mathrm{TNF}-\alpha$ at $37^{\circ} \mathrm{C}$ in an atmosphere containing $5 \% \mathrm{CO}_{2}$ for $4 \mathrm{~h}$. Resting (unactivated) HUVECs served as a control. The TCMBs or CMBs were passed across the HUVECs at a flow rate of 2 dynes $/ \mathrm{cm}^{2}$, as previously reported (19). The microbubbles that adhered to the HUVECs in the presence or absence of TNF- $\alpha$ stimulation were observed and counted.

Targeting ability of TCMBs to the human umbilical cord vein ex vivo. To test the targeting ability of the TCMBs in blood vessels, the human umbilical cord vein was used to mimic a blood vessel. Segments of the human umbilical cord vein were washed with a heparinized solution $(20 \mathrm{UI} / \mathrm{ml})$ and filled with ECM containing $50 \mathrm{ng} / \mathrm{ml} \mathrm{TNF}-\alpha$ for $6 \mathrm{~h}$ at $37^{\circ} \mathrm{C}$ to induce an inflammatory condition. Then, $2 \times 10^{8} \mathrm{TCMB}$ or $\mathrm{CMB}$ were diluted in normal saline to a total volume of $10 \mathrm{ml}$. The diluted microbubble solutions were then passed through the umbilical vein using an automatic infusion pump at $2 \mathrm{ml} / \mathrm{min}$. A $10-\mathrm{MHz}$ vascular ultrasound probe (Philips Medical Systems, Inc., Bothell, WA, USA) was used to detect the adhesion of the microbubbles to the umbilical vein following stimulation with or without TNF- $\alpha$. A video was taken, stored and analyzed using Qlab 6.0 software (Philips Medical Systems, Inc.).

Acute myocardial infarction (AMI) model. A total of 90 healthy New Zealand white rabbits (male; 4 months old; weight, $2.5 \pm 0.2 \mathrm{~kg}$ ) were provided by the Wuhan Institute of Biological Products, Co., Ltd. (Wuhan, China). Rabbits were housed at $20^{\circ} \mathrm{C}$ and $60 \%$ humidity with a $12 \mathrm{~h}$ light-dark cycle and free to access food and water.

Rabbits were anesthetized by ear vein injection of $30 \mathrm{mg} / \mathrm{kg}$ pentobarbital sodium (P3761; Sigma-Aldrich; Merck Millipore) and connected to an electrocardiogram (ECG). The heart was exposed, a left thoracotomy was performed and the left circumflex coronary artery was ligated $5 \mathrm{~mm}$ below the left atrial appendage using a 6-0 polypropylene suture. The ends of the ligature were passed through a short PE 10 tube to form a snare. Following ligation, contraction of the regional myocardium was decreased and the infarcted area turned pale. AMI was confirmed as an ST-segment elevation $>2 \mathrm{~mm}$ on the ECG. Reperfusion was induced by releasing the snare $2 \mathrm{~h}$ after AMI and was verified by a $>50 \%$ drop of the ST-segment $\geq 15$ min.

All animal experiments described in this section conformed to the Guide for the Care and Use of Laboratory Animals published by the US National Institutes of Health (NIH Publication No. 85-23, revised 1996). Approval from the Institutional Animal Care and Use Committee of Wuhan University Health Science Center was also obtained prior to performing experiments.

Imaging targeted TCMBs in the infarcted heart. To test the accumulation of TCMBs in infarcted heart, myocardial contrast echocardiography was performed as previously reported (22). Briefly, 10 rabbits with similar lengths of infarcted myocardium $(1.0-1.5 \mathrm{~cm})$ were randomly selected and $1 \mathrm{ml}$ of either TCMBs $(n=5)$ or CMBs $(n=5)$ was slowly injected for 5 min through the ear vein 2 days after AMI and reperfusion. The slow injection allowed for interaction between the microbubbles and the ICAM-1 expressed in infarcted myocardium. An S5-1 cardiac ultrasound probe (Philips Medical Systems, Inc.) was used to observe the filling of microbubbles into the myocardium. Ultrasound contrast images were acquired 3 min after the microbubbles were completely infused. The intensity of the signals originating from the retained microbubbles in the left ventricular anterior wall (infarct area) and the inferior wall (non-infarct area) was measured. The ratio of the intensity of the signals (anterior wall:inferior wall) was calculated to evaluate the adhering ability of the microbubbles to the infarcted myocardium. These rabbits were used in further experiments.

Experimental grouping. To ensure that infarcted areas in the experimental animals were all similar, rabbits with an infarcted myocardium length of $>1.5$ or $<1.0 \mathrm{~cm}$ were excluded from this study. The remaining 65 rabbits with AMI and reperfusion were randomly divided into three groups and used for gene transfection. The targeted cationic microbubble group (TCMB group; $n=25$ ) received an intravenous injection of TCMBs and the Ang-1 plasmid suspension with ultrasound exposure. The non-targeted cationic microbubble group (CMB group; $n=25$ ) received an intravenous injection of CMBs and the Ang-1 plasmid suspension with ultrasound exposure. The control group $(n=15)$ received an intravenous injection of the Ang-1 plasmid alone.

Gene transfection by ultrasound-mediated microbubble destruction. Ultrasound-targeted microbubble destruction-mediated gene transfection was performed 2 days after AMI. The concentrations of the TCMB and CMB suspensions were adjusted to $\sim 2 \times 10^{8}$ microbubbles $/ \mathrm{ml}$. A total of $1 \mathrm{ml}$ microbubble suspension was mixed with $1 \mathrm{ml}$ Ang-1 plasmid $(1 \mathrm{mg} / \mathrm{ml})$ and the mixture was incubated at room temperature for $15 \mathrm{~min}$ to ensure sufficient contact between the plasmid and the microbubbles. Rabbits were then injected with $2 \mathrm{ml}$ plasmid-microbubble solution via the ear vein and ultrasound irradiation was performed for gene transfection. 
The iE33 ultrasound diagnostic system with an M3S transducer (Philips Medical Systems, Inc.) was used for ultrasound exposure. The 'contrast' procedure was selected and the second harmonic mode, with a frame rate of $80 \mathrm{~Hz}$, was switched on. The probe emission frequency and the receiving frequency were 1.7 and $3.4 \mathrm{MHz}$, respectively. An ECG trigger was performed every $4-8$ cardiac cycles and the depth was set at $5 \mathrm{~cm}$. Once the microbubble infusion began, the ultrasound beam was continuously aimed from the chest wall towards the heart of the rabbit. The ultrasound blasting function was activated to disrupt the microbubbles when the myocardial filling of the contrast agent reached a plateau $(\sim 30 \mathrm{sec}$ after vein injection), allowing for adhesion of the microbubbles. The ultrasound exposure (mechanical index 1.3 and 100\% overall gain) lasted for $5 \mathrm{~min}$. One flash per six cardiac cycles was used to disrupt the microbubbles and a pulsing interval allowed replenishment and adhesion of the microbubbles before the next flash, as previously reported (22).

Myocardial Ang-1 expression. Rabbits were sacrificed by intravenous injection of $30 \mathrm{ml}$ air following anesthesia with $30 \mathrm{mg} / \mathrm{kg}$ pentobarbital sodium. A total of 5 rabbits were randomly selected from each group and the hearts were harvested 3 days following transfection. The myocardial tissue from the infarct and border region was cut into small blocks of equal size $(0.5 \times 0.5 \times 0.5 \mathrm{~cm})$ and snap-frozen for the analysis of Ang-1 expression.

Semi-quantitative reverse transcription-polymerase chain reaction (RT-PCR) was used to detect myocardial Ang-1 mRNA expression in all of the groups. The primers for Ang-1 were as follows: Forward, 5'-TGCCATTACCAGTCAGAGG-3' and reverse, 5'-CAAGCATCAAACCACCATC-3'; the primers for rabbit $\beta$-actin were as follows: Forward, 5'-GTGCTTCTA GGCGGACTGTTAGA-3' and reverse, 5'-CACGAATAA AGCCATGCCAAT-3'. Total RNA was extracted from $100 \mathrm{mg}$ myocardium using TRIzol reagent according to the manufacturer's protocol (15596-026; Invitrogen; Thermo Fisher Scientific, Inc.). The samples were then treated with DNase I (18068-015; Invitrogen; Thermo Fisher Scientific, Inc.) for 15 min at room temperature, and the RNA was further purified using an RNA cleanup kit (74204; Qiagen, Inc., Valencia, CA, USA). cDNA was synthesized using SuperScript III First-Strand Synthesis System RT-PCR kit (18080-051; Invitrogen; Thermo Fisher Scientific, Inc.), according to the manufacturer's protocol. PCR was performed with a final reaction volume of $50 \mu \mathrm{l}$ containing PCR amplification buffer (1X), Taq DNA polymerase (2.5 U), dNTPs (4 mM), primers $(0.4 \mu \mathrm{M})$ and template DNA (4 ng). Cycling conditions were as follows: $94^{\circ} \mathrm{C}$ for $2 \mathrm{~min}$, followed by 30 cycles of $94^{\circ} \mathrm{C}$ for $30 \mathrm{sec}, 55^{\circ} \mathrm{C}$ for $30 \mathrm{sec}, 72^{\circ} \mathrm{C}$ for $1 \mathrm{~min}$, and a final extension step at $72^{\circ} \mathrm{C}$ for $5 \mathrm{~min}$. PCR products were electrophoresed on a $1 \%$ agarose gel and stained with ethidium bromide. The relative expression of Ang-1 mRNA was normalized to that of $\beta$-actin using a gel imaging analysis system (Geliance 200; PerkinElmer, Inc., Waltham, MA, USA).

Western blot analysis was performed as described above to determine the Ang-1 protein expression in the myocardium from the infarcted and border regions, and the relative expression of the Ang-1 protein was normalized to that of $\beta$-actin. The primary antibody used was rat anti-rabbit Ang-1 polyclonal antibody (1:200; bs-0800R; Beijing Biosynthesis Biotechnology Co., Ltd.) and the secondary antibody was horseradish peroxidase-coupled goat anti-rat $\operatorname{IgG}(1: 2,000$; ab97057; Abcam).

Cardiac function. A standard echocardiography examination was performed in all groups the day before AMI, 2 days after AMI and 4 weeks following gene transfection. The left ventricular morphology and motion were observed and the left ventricular end-diastolic and end-systolic dimensions (LVEDD and LVESD, respectively), left ventricular ejection fraction (LVEF) and left ventricular fractional shortening (LVFS) were measured to assess changes in left ventricular function.

Myocardial perfusion. Myocardial contrast echocardiography (MCE) was performed 4 weeks after gene transfection. A total of $1 \mathrm{ml} \mathrm{SonoVue}{ }^{\circledR}$ microbubble solution (Bracco SpA, Milan, Italy) was injected via the ear vein and the short-axis view at the papillary muscle level was obtained to assess myocardial perfusion. All acquired images were transferred to the workstation for a frame-by-frame, off-line analysis. The myocardial blood volume was analyzed by calculating the ratio of the intensity of the contrast agent in the left ventricular anterior wall (infarct area) to that in the inferior wall (non-infarct area) in the digital images. The MCE images were analyzed using Qlab 6.0 software.

Angiogenesis. Myocardium from the infarct and border regions was obtained 4 weeks after gene transfection and immunohistochemical staining for von Willebrand Factor (vWF) was performed. Sections of rabbit myocardium from the infarcted and border regions were fixed with $4 \%$ paraformaldehyde at room temperature for $24 \mathrm{~h}$ and embedded in paraffin. The sections were incubated in a citrate buffer $(0.01 \mathrm{M} ; \mathrm{pH} 6.0$; P0081; Beyotime Institute of Biotechnology) at $100^{\circ} \mathrm{C}$ for $15 \mathrm{~min}$ for antigen retrieval. The sections were then blocked in blocking buffer (5\% normal goat serum; C0265; Beyotime Institute of Biotechnology) at $37^{\circ} \mathrm{C}$ for $20 \mathrm{~min}$ and incubated with a rabbit anti-vWF antibody (1:200; bs-10048R, Beijing Biosynthesis Biotechnology Co., Ltd.) overnight at $4^{\circ} \mathrm{C}$. Sections were subsequently incubated with biotin conjugated secondary antibody (goat anti-rabbit IgG; 1:200; bs-0295G-Bio, Beijing Biosynthesis Biotechnology Co., Ltd.) for $40 \mathrm{~min}$ and treated with streptavidin peroxidase for $10 \mathrm{~min}$ at $37^{\circ} \mathrm{C}$ Finally, specimens were incubated in DAB for 5 mins at $37^{\circ} \mathrm{C}$. The microvascular density (MVD) was then determined using optical microscopy (x200) according to the Weidner criteria (23). A pathologist was employed to average the five visual fields with the most capillaries in single-blind manner.

Histomorphometry. At 4 weeks after transfection, rabbit hearts were harvested, fixed at $37^{\circ} \mathrm{C}$ overnight with $10 \%$ paraformaldehyde and sectioned. The sections (thickness, $5 \mu \mathrm{m}$ ) were stained with Masson's trichrome stain to assess the morphology of the heart, infarct size and collagen deposition in the myocardium.

Statistical analysis. Data are expressed as the mean \pm standard deviation and were analyzed using GraphPad Prism 5 software 
A

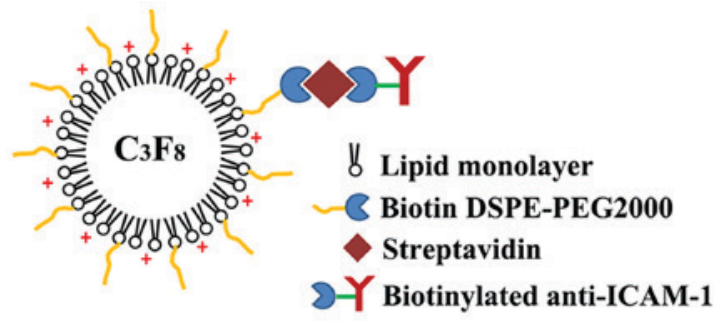

B

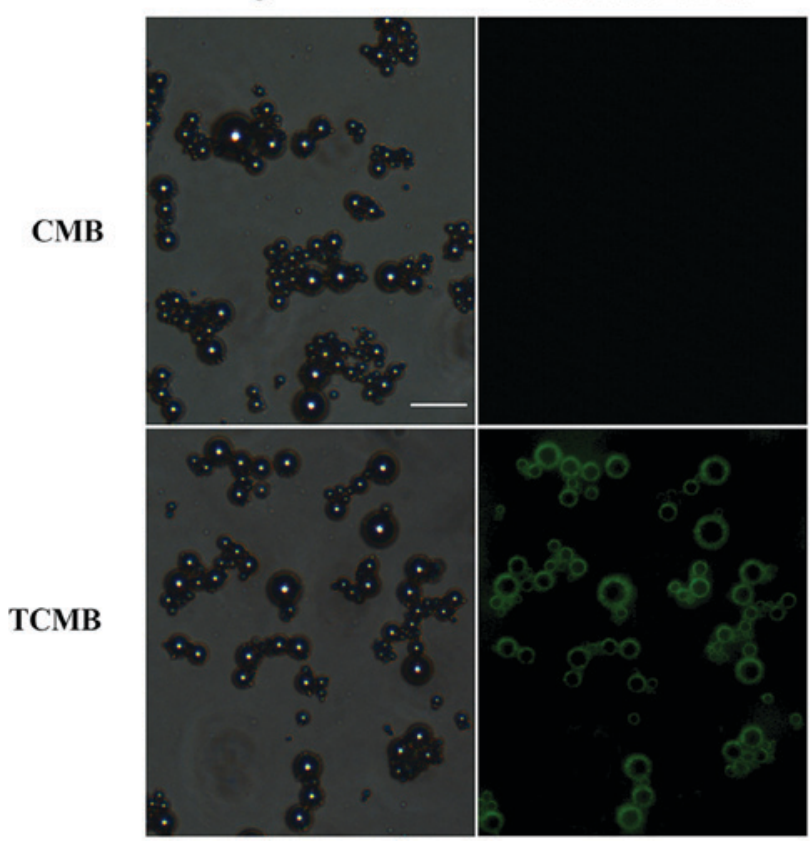

Figure 1. (A) Schematic illustration of a TCMB. An ICAM-1 antibody was conjugated to the microbubbles using biotin-streptavidin bridging chemistry. (B) CMBs and TCMBs under light and fluorescence microscopy (scale bar, $15 \mu \mathrm{m}$ ). The green fluorescent ring around the microbubble surface indicates the combination of the fluorescein isothiocyanate-labeled immunoglobulin $\mathrm{G}$ and the ICAM-1 antibody on the surface of a cationic microbubble. ICAM-1, intercellular adhesion molecule-1; TCMB, targeted cationic microbubble; CMB, non-targeted cationic microbubbles; DSPE-PEG2000, 1,2-distearoyl-sn-glycero-3-phosphoethanolamine- $\mathrm{N}$-[maleimide (polyethylene glycol)-2000].

(GraphPad Software, Inc., La Jolla, CA, USA). Differences between groups prior to and following gene transfection were analyzed using paired $t$-tests. Comparisons among multiple groups were made using a one-way analysis of variance with Student Newman Keuls-q post hoc analysis. All statistical analyses were two-sided and $\mathrm{P}<0.05$ was considered to indicate a statistically significant difference.

\section{Results}

Characterization of TCMBs and DNA conjugation. A schematic of a TCMB is presented in Fig. 1A. The microbubbles were observed to be smooth and spherical. Fig. 1B shows the microscopic morphology of the CMBs and TCMBs under bright-field and fluorescence microscopy, respectively. Bright green fluorescence was present at the periphery of each microbubble, which indicated that the ICAM-1 antibodies were successfully bound to the surface of the microbubbles. The average diameters of the TCMBs and CMBs were 3.1 and
A

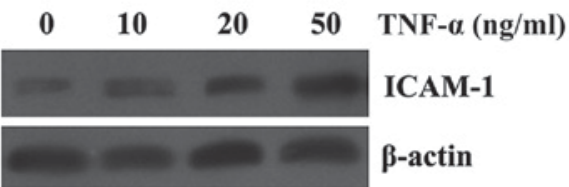

B

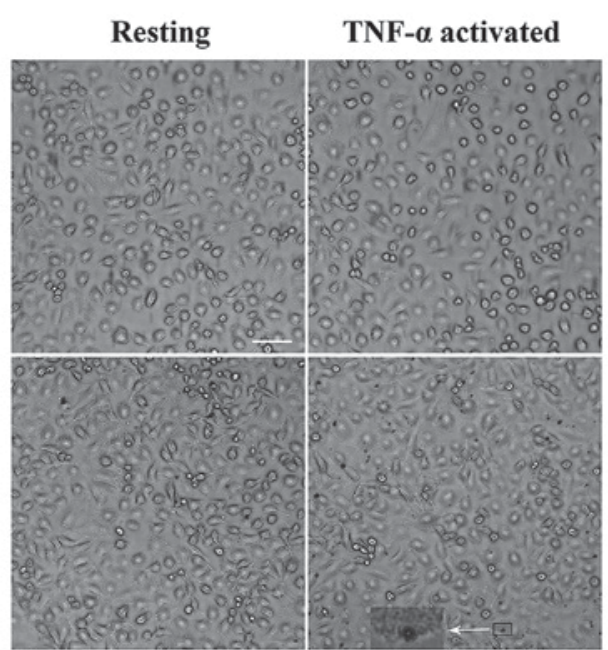

C

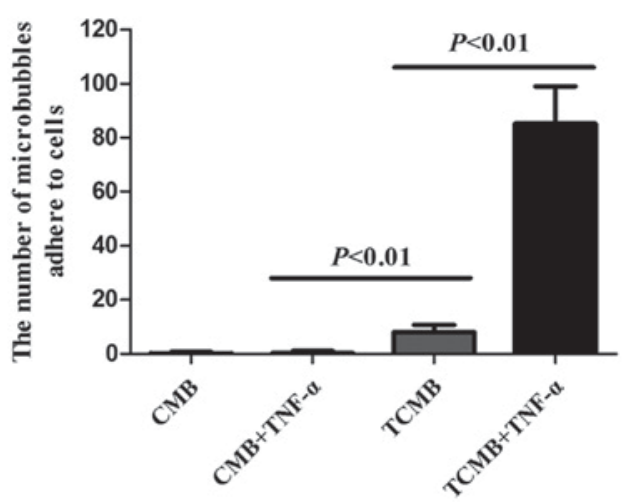

Figure 2. Microbubbles targeted to HUVECs in vitro. (A) ICAM-1 protein expression in HUVECs activated by various doses of TNF- $\alpha(0,10,20$ and $50 \mathrm{ng} / \mathrm{ml}$ ). (B) Representative images of CMBs and TCMB0s attached to resting and TNF- $\alpha$-activated HUVECs. The inset shows a high-magnification view of a microbubble attached to a HUVEC. Magnification, x400; scale bar, $150 \mu \mathrm{m}$. (C) Number of TCMBs adhered to HUVECs was 18.4-fold greater than that of CMBs following TNF- $\alpha$ stimulation ( $85 \pm 14$ vs. $8 \pm 3$ microbubbles per microscopic field). HUVECs, human umbilical vein endothelial cells; TNF, tumor necrosis factor; CMB, non-targeted cationic microbubble; TCMB, targeted cationic microbubble; ICAM-1, intracellular adhesion molecule-1.

$2.9 \mu \mathrm{m}$, respectively and there were no significant differences between groups $(\mathrm{P}>0.05)$. The zeta potentials of the TCMBs and CMBs were $+22.0 \pm 2.4$ and $+31.3 \pm 3.9 \mathrm{mV}(\mathrm{P}<0.01)$. This difference most likely resulted from the conjugation of the positively charged ICAM-1 antibodies on the surface of the TCMBs. UV spectrophotometric analysis indicated that the DNA-binding capacity of the TCMBs was mildly reduced compared with that of the CMBs $(3.3 \pm 0.7$ and $4.0 \pm 0.9 \mu \mathrm{g}$ $10^{8} /$ microbubble; $\left.\mathrm{P}<0.05\right)$.

Targeting ability of TCMBs to inflammatory endothelial cells in vitro. The expression of ICAM-1 in inflammatory endothelial cells was analyzed using western blotting. The expression of ICAM-1 in HUVECs was upregulated in a dose-dependent manner following TNF- $\alpha$ stimulation (Fig. 2A). 
A

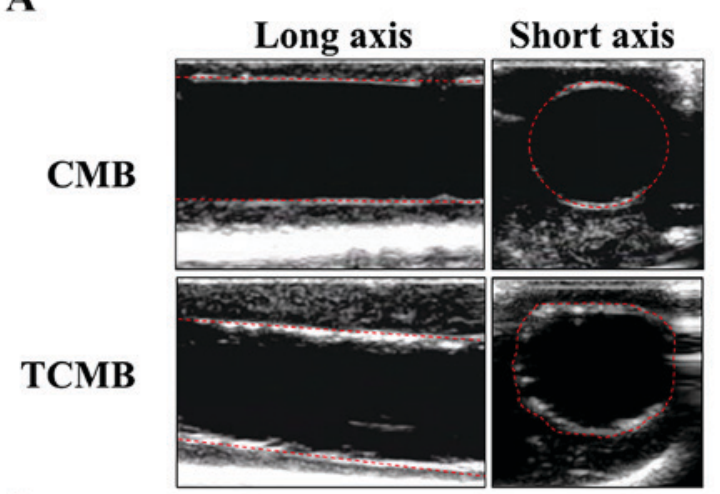

B

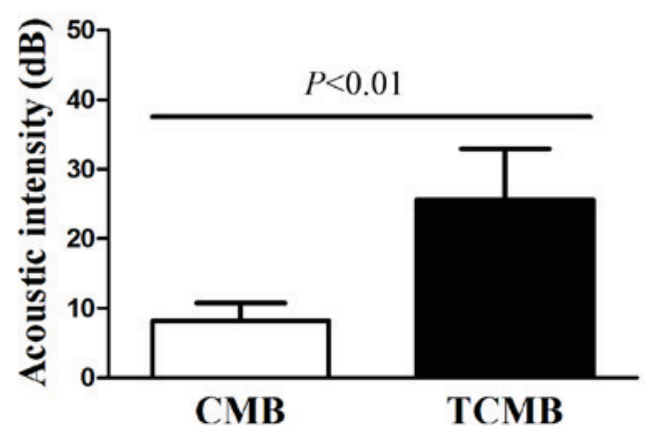

Figure 3. Ultrasonic analysis of microbubble-specific targeting to human umbilical cord veins. (A) Representative images of CMBs and TCMBs attached to human umbilical cord veins following TNF- $\alpha$ stimulation. The red dashed lines delineate the intima of the umbilical vein. A greater number of TCMBs attached to the intima of the human umbilical vein that was activated by TNF- $\alpha$. (B) Acoustic intensity of the TCMBs and CMBs adhered to the umbilical vein endothelium was $25.6 \pm 7.3$ and $8.2 \pm 2.6 \mathrm{~dB}$, respectively. $\mathrm{CMB}$, non-targeted cationic microbubble; TCMB, targeted cationic microbubble; TNF, tumor necrosis factor.

The flow chamber experiment showed that a large number of TCMBs adhered to HUVECs following TNF- $\alpha$ stimulation, whereas no significant adhesion occurred in the resting condition. Regardless of whether the cells were stimulated with TNF- $\alpha$ or not, there was no significant adhesion of CMBs to the HUVECs (Fig. 2B). However, data from microbubble counting indicated that the number of adhered TCMBs was 18.4-fold greater than that of CMBs following TNF- $\alpha$ stimulation $(85 \pm 14$ vs. $8 \pm 3$ microbubbles per microscopic field, $\mathrm{P}<0.001$; Fig. $2 \mathrm{C}$ ).

Targeting ability of TCMBs to the human umbilical cord vein ex vivo. The flow chamber experiment confirmed that TCMBs adhered to HUVEC membranes under controlled physiological conditions. Subsequently, the umbilical cord vein was used to simulate a vessel and further evaluate the targeted adhesion of the TCMBs to the vascular endothelium. CMBs and TCMBs were visible in the ultrasound field and there were no significant differences in the visibility of the groups. The results demonstrated that TCMBs were able to adhere to TNF- $\alpha$-stimulated umbilical vein endothelium under flow conditions but did not adhere notably in the resting condition. No significant CMB adhesion to the umbilical vein was observed in TNF- $\alpha$-stimulated or resting conditions, which eliminated the possibility of nonspecific adhesion. Acoustic quantitative analysis showed that the acoustic intensity of the
A

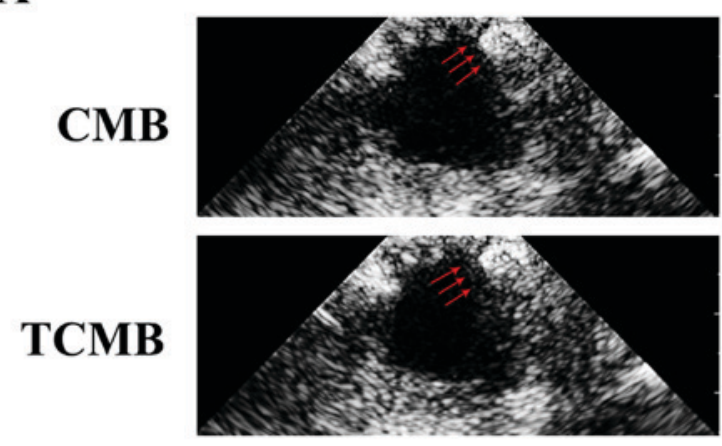

B

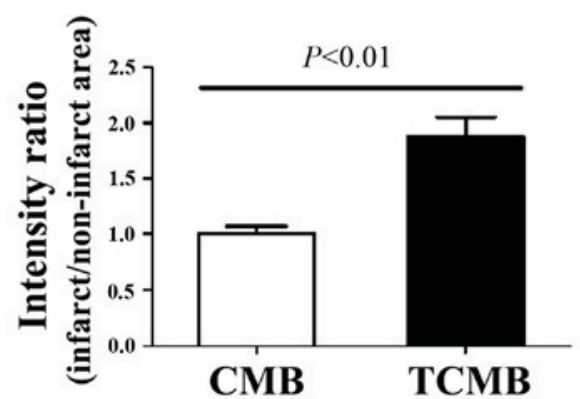

Figure 4. Targeted microbubbles adhere to the infarcted zone. (A) Representative images of the CMBs and TCMBs that accumulated within the infarcted region 3 min after intravenous injection. Red arrows indicate the infarcted area. (B) Compared with the CMB group, the targeting ability of the TCMBs to the infarcted zone was significantly greater $(n=5$ per group). $\mathrm{CMB}$, non-targeted cationic microbubble; TCMB, targeted cationic microbubble.

TCMBs adhered to the umbilical vein endothelium was significantly higher than that of the CMBs $(25.6 \pm 7.3$ vs. $8.2 \pm 2.6 \mathrm{~dB}$, $\mathrm{P}<0.001$; Fig. 3).

Targeting ability of TCMBs to infarcted myocardium in vivo. To evaluate the ability of the microbubbles to target infarcted tissue, ultrasound imaging was performed to determine microbubble intensity in the left ventricle 3 min following intravenous injection. In the CMB group, no microbubble accumulation was detected in the infarcted region. By comparison, intense contrast enhancement was observed in the TCMB group (Fig. 4A). The ratio of the signal intensity (anterior wall/inferior wall) in the TCMB group was $86 \%$ greater than that of the CMB group $(\mathrm{P}<0.01$; Fig. $4 \mathrm{~B})$. These results demonstrate that the TCMBs were able to adhere at the infarcted site and were retained.

Ultrasound-mediated TCMB destruction increases Ang-1 gene expression in ischemic myocardium. RT-PCR and western blot analyses were conducted to evaluate the delivery of Ang-1 to the infarcted myocardium 3 days following UTMD-mediated gene transfection. The results indicated that the expression of Ang-1 mRNA in the TCMB group was significantly increased compared with that in the CMB group $(\mathrm{P}<0.01)$ and very low Ang-1 mRNA expression was detected in the controls (Fig. 5A).

Results from the western blot analysis demonstrated that the expression of Ang-1 protein in the TCMB group was 
A
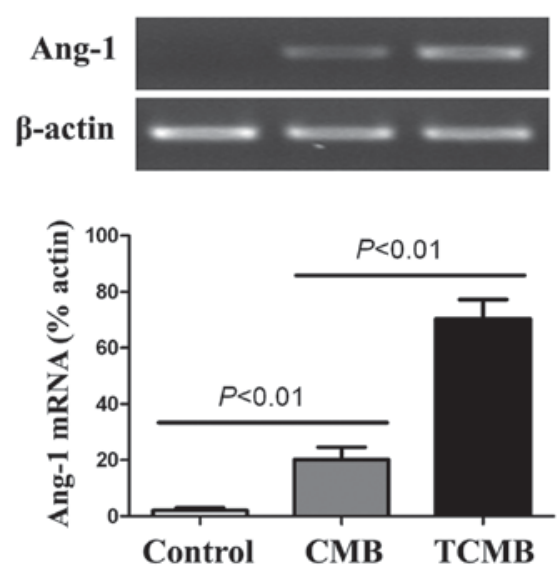

B
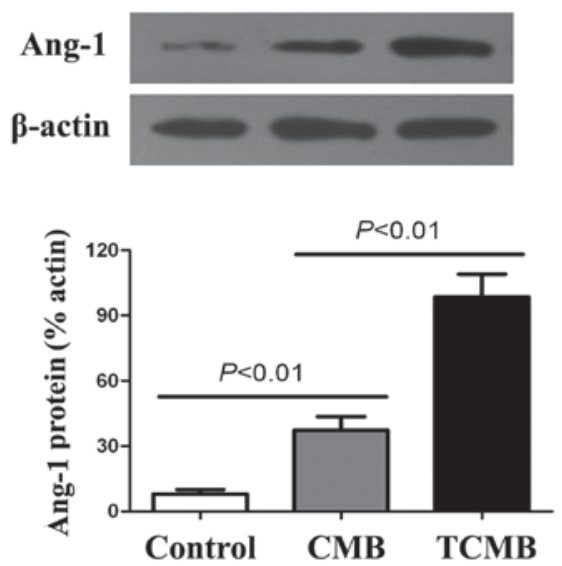

Figure 5. Targeted Ang-1 gene expression. (A) Semi-quantitiative reverse transcription polymerase chain reaction and (B) western blot analyses were conducted to detect Ang-1 mRNA and protein expression, respectively, 3 days after ultrasound-targeted microbubble destruction-mediated Ang-1 gene delivery. The highest levels of Ang-1 mRNA and protein expression were observed in the TCMB group (n=5/group). Ang-1, angiopoietin 1; CMB, non-targeted cationic microbubble; TCMB, targeted cationic microbubble.

$\mathbf{A}$

Control

CMB

TCMB

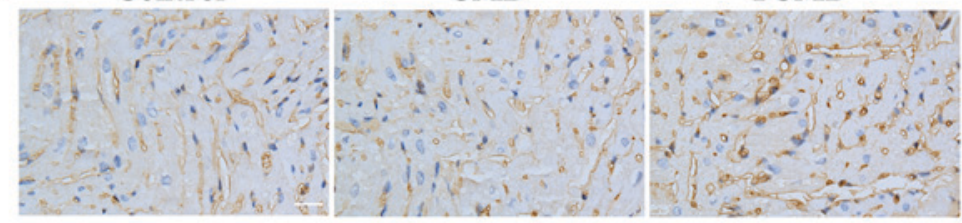

B

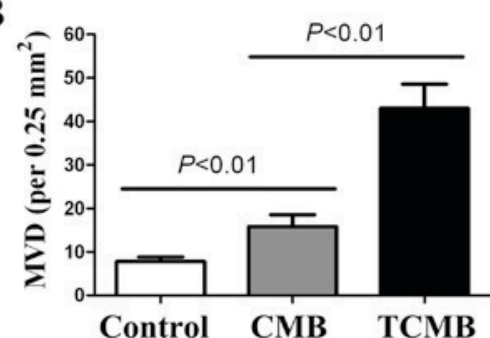

C

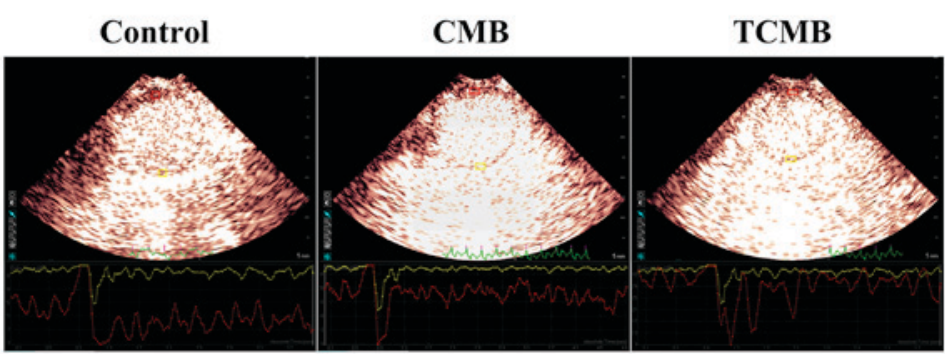

D

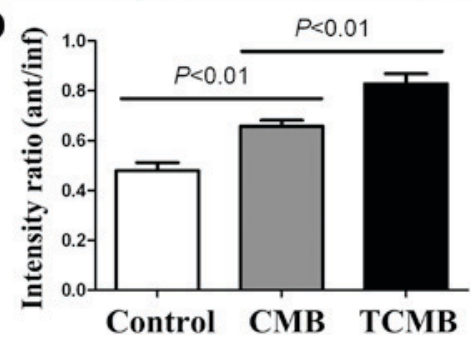

Figure 6. Angiogenesis and myocardial perfusion in infarcted hearts. (A) Neovascularization of the infarct and border areas was detected following immunostaining for von Willebrand factor. The number of capillaries was counted to calculate the microvascular density (scale bar, $50 \mu \mathrm{m}$ ). (B) MVD in the TCMB group was significantly higher than that of the CMB and the control groups. (C) Myocardial contrast echocardiography was performed to assess the myocardial perfusion [presented as the ratio of the signal intensity in the anterior wall (infarct area) to that in the inferior wall (non-infarct area)] in the infarct area. (D) Compared with the CMB and control groups, ultrasound-targeted microbubble destruction-mediated Ang-1 gene delivery significantly improved myocardial perfusion in the TCMB group ( $n=20$ in each of the TCMB and CMB groups; $n=10$ in the control group). MVD, microvascular density; TCMB, targeted cationic microbubble; $\mathrm{CMB}$, non-targeted cationic microbubble. 


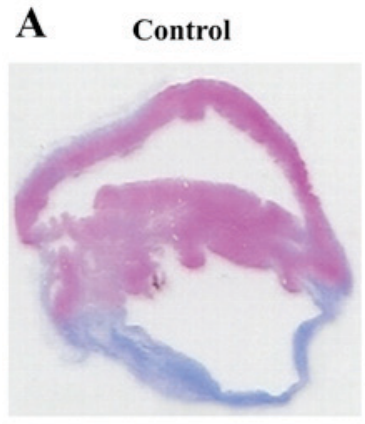

B

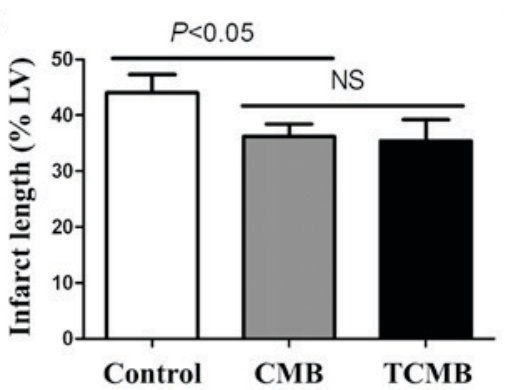

CMB

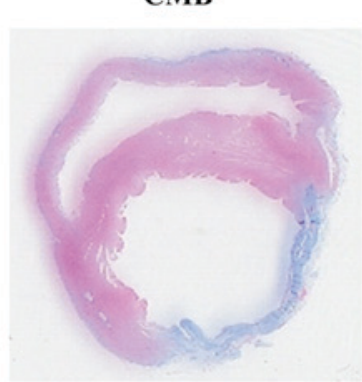

TCMB

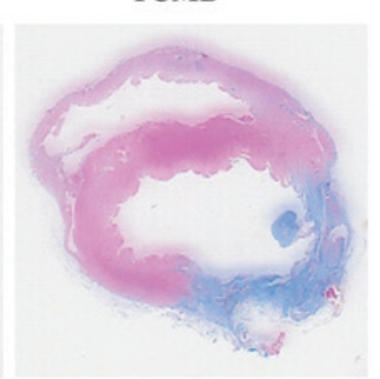

C

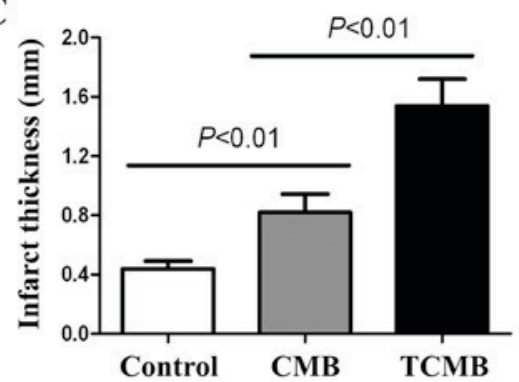

Figure 7. Histomorphology of infarcted hearts. (A) Representative histological Masson's trichrome staining of transverse sections of infarcted hearts 28 days following UTMD-mediated Ang-1 gene delivery. The infarct length and thickness (blue) of the left ventricular wall are presented (no magnification). The (B) infarct length and (C) thickness of the left ventricular wall were measured 4 weeks after UTMD-mediated Ang-1 gene delivery. The TCMB and CMB groups ( $\mathrm{n}=20$ per group) had similar infarct lengths, which were significantly lower than that of the control group. However, the thickness of the infarct region was significantly increased in the TCMB group compared with that of the CMB and control group ( $\mathrm{n}=10$ in the control group). Ang-1, angiopoietin 1; UTMB, ultrasound-targeted microbubble destruction; $\mathrm{CMB}$, non-targeted cationic microbubble; TCMB, targeted cationic microbubble; NS, not significant.

significantly higher than in the CMB group $(\mathrm{P}<0.01)$. Additionally, very low Ang-1 protein expression detected in the controls (Fig. 5B).

Ultrasound/ICAM-1-targeted, microbubble-mediated Ang-1 gene transfection increases myocardial angiogenesis and improves perfusion. At 4 weeks after gene transfection, immunohistochemical staining for vWF was performed to determine the microvascular density (MVD) in the infarct and border regions. The results indicated that the MVD in the infarct and border regions (primarily at the border region of the infarcted myocardium) of the TCMB group was significantly increased compared with that of the $\mathrm{CMB}$ and control groups (all $\mathrm{P}<0.01$; Fig. 6A and B).

MCE demonstrated that the contrast agent was markedly absent from the anterior wall; however satisfactory filling occurred 2 days following AMI in other regions. The ratios of the signal intensity in the anterior wall (infarct region) to that of the inferior wall (non-infarct region) of the three groups were low and similar to each other (data not shown). A total of 4 weeks after transfection, the perfusion of the infarcted myocardium was improved in all groups; however, the improvement in the TCMB group was greater than that in the $\mathrm{CMB}$ group, which in turn, was greater than that in the control group (all $\mathrm{P}<0.01$; Fig. $6 \mathrm{C}$ and $\mathrm{D}$ ).

Ultrasound/ICAM-1-targeted, microbubble-mediated Ang-1 gene transfection improves cardiac morphology and function. Masson's trichrome staining demonstrated that the rabbits in the control group had the largest left ventricle, the largest range of infarction, the thinnest ventricular wall and the greatest collagen deposition in the infarct and border myocardia.
Rabbits in the CMB group had a thicker ventricular wall and a smaller left ventricle, a smaller range of infarction and collagen deposition, and the TCMB group had the smallest and thickest infarct wall compared with the control group (Fig. 7A). The TCMB group had a similar infarct length to the CMB group; however, the infarct lengths in both microbubble groups were significantly smaller than that of the control group $(\mathrm{P}<0.05$; Fig. 7B). However, the TCMB group had a significantly greater infarct thickness than the $\mathrm{CMB}$ and control groups $(\mathrm{P}<0.01$; Fig. 7C) These data demonstrated that Ang-1 gene transfection mediated by the ultrasound destruction of TCMBs prevented the infarcted region from thinning and extending and improved ventricular remodeling following infarction (Fig. 7).

At 2 days after AMI, left ventricular anterior wall motion and ejection fraction were significantly reduced in all groups and there were no significant differences among the three groups. Additionally, 4 weeks following Ang-1 gene transfection, LVEDD decreased in the CMB and TCMB groups compared with the baseline dimensions, while the LVEF and LVFS increased by various degrees in all groups with the exception of the control group. The greatest improvements in LVEDD, LVEF and LVFS occurred in the TCMB group (Fig. 8).

\section{Discussion}

The aim of the present study was to test the hypothesis that cationic microbubbles conjugated to an ICAM-1 antibody by biotin-streptavidin chemical conjugation could bind to and target the delivery of the Ang-1 gene to the infarcted heart, thus improving cardiac function. The results of the current study indicated that TCMBs were able to recognize injured vascular 

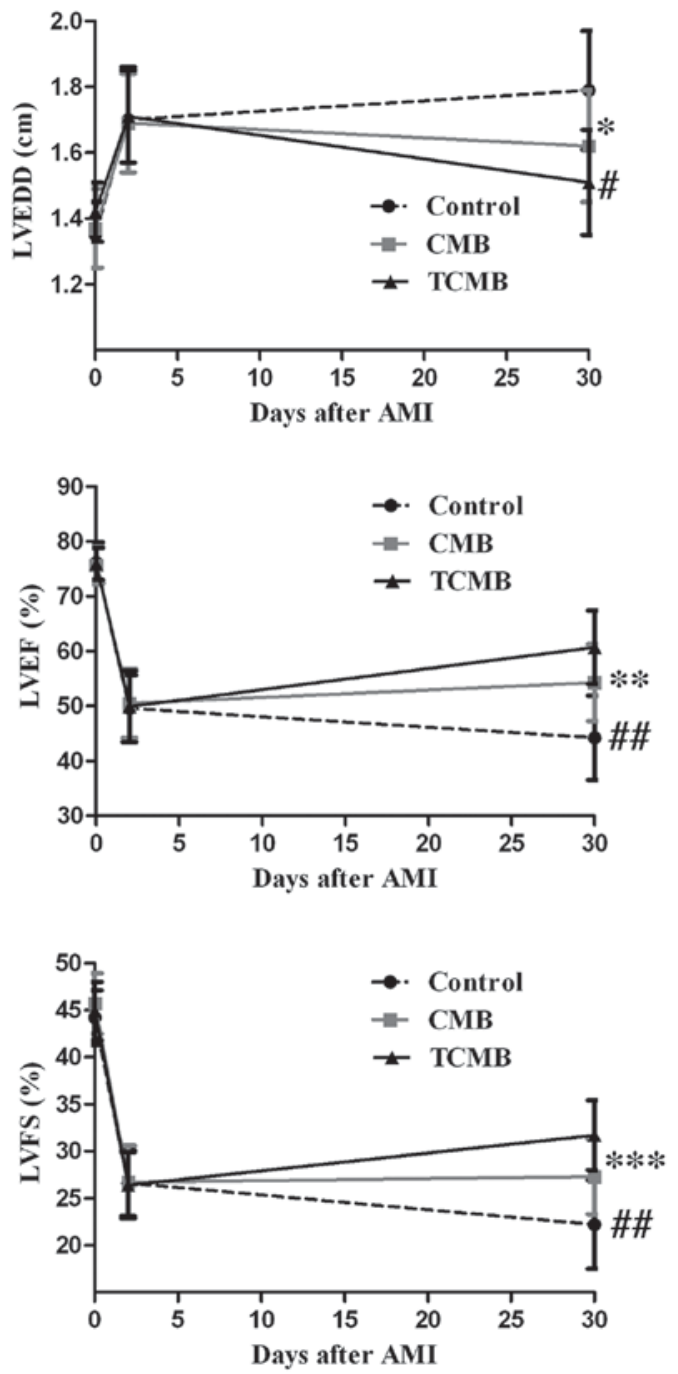

Figure 8. Echocardiographic assessment of cardiac function. Compared with the control group $(n=10)$, LVEDD, LVEF and LVFS in the CMB group $(n=20)$ were slightly improved 4 weeks following ultrasound-targeted microbubble destruction-mediated angiopoietin 1 gene delivery; however, a greater improvement was observed in the TCMB group $(\mathrm{n}=20)$. $\mathrm{P}=0.001,{ }^{* *} \mathrm{P}=0.034$ ${ }^{* * * *} \mathrm{P}=0.025$ vs. control; ${ }^{~} \mathrm{P}=0.04,{ }^{\# \#} \mathrm{P}=0.001$ vs. $\mathrm{CMB}$. LVEDD, left ventricular end-diastolic dimension; LVEF, left ventricular ejection fraction; LVFS, left ventricular fractional shortening; $\mathrm{CMB}$, non-targeted cationic microbubble TCMB, targeted cationic microbubble.

endothelium and site-specifically deliver the Ang-1 gene to the infarct area following myocardial infarction. Compared with the non-targeted microbubbles, TCMBs significantly increased the efficiency of UTMD-mediated Ang-1 gene transfection in the myocardium of the infarcted and border regions. High expression of Ang-1 enhanced angiogenesis, improved cardiac perfusion and promoted myocardial repair.

UTMD is a promising strategy for gene delivery due to its hypotoxicity, low immunogenicity and reproducibility of use (8-11). However, the therapeutic effects of UTMD have been somewhat limited as the efficiency of gene transfection in vivo remains unsatisfactory (24). A number of factors, including the ultrasound parameters, exposure equipment, the microbubble characteristics and the types of target tissues and cells that are transfected, affect the transfection efficiency of UTMD, and gene concentration at the site of sonoporation is a critical factor for gene transfection in vivo $(25,26)$. There are two factors that determine the gene concentration at the site of sonoporation: The gene-binding capacity of the microbubbles and the targeted adhesion of the microbubbles to the surface of the cells.

The gene-binding capacity of microbubbles is a key factor affecting the concentration of genes at the site of sonoporation (27). Previous studies used commercially available microbubbles, including SonoVue or Definity microbubbles, as in vivo gene delivery vehicles $(28,29)$. In these studies, plasmid DNA was mixed and incubated with microbubbles to couple the genes to the microbubbles prior to intravenous injection. This direct coupling strategy was simple to perform; however, the negatively charged surfaces of the commercially available microbubbles prevented their effective conjugation to the negatively charged nucleic acid due to the repulsion of the electric charges. The unconjugated plasmid DNA was cleared and degraded by endogenous nucleases prior to reaching the target tissue (30). In the present study, a cationic microbubble composed of DC-Chol, DSPC, DSPE-PEG and DPPA was synthesized. DC-Chol is a well-known cationic lipid that can provide a positive surface charge for microbubbles (31). The other lipids were used to form the components of the lipid shell and to increase the stability of the microbubbles in blood. The results of the current study demonstrated that the cationic microbubble had a significant positive zeta potential $(+31.3 \pm 3.9 \mathrm{mV})$ and the combination of the plasmid DNA and the cationic microbubbles was as high as $4 \mu \mathrm{g} / 10^{8}$ microbubbles in a neutral $\mathrm{pH}$ environment. Furthermore, although the ICAM-1 antibody was conjugated to the cationic microbubbles, the microbubbles showed a net positive charge $(+22.0 \pm 2.4 \mathrm{mV})$. The cationic microbubbles were able to form stable complexes with the plasmid DNA by electrostatic attraction and they protected the plasmid DNA from degradation by endogenous nucleases (32). This may produce an obvious advantage when used in vivo.

An alternative approach to increase the concentration of genes at the site of sonoporation is to specifically target microbubbles to the issue of interest. Non-targeted microbubbles may disperse throughout the entire body, resulting in unsatisfactory treatment even though the operator-guided ultrasound destruction of the microbubbles releases the gene with a certain degree of targeting. Previous studies have described the successful coupling of specific ligands to microbubbles, thereby making it possible for microbubbles to recognize a target tissue $(33,34)$. Wang et al (33) constructed a phospholipid microbubble conjugated to a single-chain antibody specific for activated glycoprotein IIb/IIIa, due to its binding to a ligand-induced binding site; the results demonstrated that the targeting microbubble may be used to detect atherosclerotic plaques. Leong-Poi et al (35) prepared $\alpha \mathrm{V}$-integrin-targeted microbubbles by conjugating a monoclonal antibody against the $\alpha \mathrm{V}$ integrin to the microbubble surface. The targeted microbubbles directly attached to the microvascular endothelial surface, thus allowing assessment of the treatment as a promoter of therapeutic angiogenesis (35). In the present study, a cationic microbubble conjugated to an ICAM-1 antibody was constructed. ICAM is widely distributed on the surface of many cells, such as endothelial cells. ICAM expression is quite low under normal conditions; however, its expression is significantly increased following the activation of endothelial cells by inflammatory cytokines, ischemia or 
hypoxia $(36,37)$. The upregulation of ICAM-1 expression is an important marker of endothelial cell injury and leukocyte activation (16). Following the occurrence of ischemia, multiple inflammatory factors induce the overexpression of ICAM-1 in microvascular endothelial cells $(38,39)$. Therefore, ICAM-1 is a potential marker for early detection of inflammation/injury and may be a possible target for gene delivery. The results of the current study demonstrated that microbubbles conjugated to ICAM-1 antibodies were able to recognize inflammatory vascular endothelial cells and deliver a greater concentration of the Ang-1 gene to the ischemic myocardium through their selective adhesion to the injured endothelium. Flow chamber studies indicated that the coupling of Ang-1 plasmid DNA to the surface did not interfere with the targeting of the TCMBs. Compared with the CMBs, the number of TCMBs that adhered to the cells was 18.4-fold greater following TNF- $\alpha$ stimulation in vitro. Furthermore, the number of TCMBs that adhered to the umbilical vein endothelium was significantly increased compared with the number of adhered CMBs. Additionally, a large number of TCMBs were specifically retained in and adhered to the injured vascular endothelium of the infarcted rabbit myocardium, whereas no CMBs adhered to the infarcted myocardium. The specific adhesion of microbubbles carrying a gene to a site of injured vascular endothelium may improve contact between the gene and the endothelium and increase the amount of the gene delivered to the target tissue. The results of the current study showed that the level of Ang-1 gene expression and the subsequent biological effects, including changes in the morphology and function of the infarct heart and myocardial perfusion, were all significantly improved in the TCMB group compared with the CMB group.

In conclusion, the data collected in the present study data demonstrate that ICAM-1-targeted cationic microbubbles are able to effectively carry the Ang-1 gene. They specifically accumulate at the site of injury following myocardial infarction and therefore may be a promising carrier for gene therapy. The use of ultrasound and ICAM-1-targeted cationic microbubbles to promote angiogenesis using gene therapy is a feasible, simple and effective approach that may have clinical applications in the treatment of refractory ischemic heart diseases.

\section{Acknowledgements}

The present study was funded by the National Natural Science Foundation of China (grant nos. 81471674 and 81501495).

\section{References}

1. Henry TD, Satran D, Hodges JS, Johnson RK, Poulose AK, Campbell AR, Garberich RF, Bart BA, Olson RE, Boisjolie CR, et al: Long-term survival in patients with refractory angina. Eur Heart J 34: 2683-2688, 2013.

2. Lassaletta AD, Chu LM and Sel FW: Therapeutic neovascularization for coronary disease: Current state and future prospects. Basic Res Cardiol 106: 897-909, 2011.

3. Cochain C, Channon KM and Silvestre JS: Angiogenesis in the infarcted myocardium. Antioxid Redox Signal 18: 1100-1113, 2013.

4. Katz MG, Fargnoli AS, Williams RD and Bridges CR: The road ahead: Working towards effective clinical translation of myocardial gene therapies. Ther Deliv 5: 39-51, 2014.

5. Katz MG, Fargnoli AS, Pritchette LA and Bridges CR: Gene delivery technologies for cardiac applications. Gene Ther 19: 659-669, 2012
6. Cool SK, Geers B, Lentacker I, De Smedt SC and Sanders NN: Enhancing nucleic acid delivery with ultrasound and microbubbles. Methods Mol Biol 948: 195-204, 2013.

7. Geis NA, Katus HA and Bekeredjian R: Microbubbles as a vehicle for gene and drug delivery: Current clinical implications and future perspectives. Curr Pharm Des 18: 2166-2183, 2012.

8. Ma J, DU LF, Chen M, Wang HH, Xing LX, Jing LF and Li YH: Drug-loaded nano-microcapsules delivery system mediated by ultrasound-targeted microbubble destruction: A promising therapy method. Biomed Rep 1: 506-510, 2013.

9. Smith AH, Fujii H, Kuliszewski MA and Leong-Poi H: Contrast ultrasound and targeted microbubbles: Diagnostic and therapeutic applications for angiogenesis. J Cardiovasc Transl Res 4: 404-415, 2011.

10. Liao YY, Chen ZY, Wang YX, Lin Y, Yang F and Zhou QL: New progress in angiogenesis therapy of cardiovascular disease by ultrasound targeted microbubble destruction. Biomed Res Int 2014: 872984, 2014.

11. Fujii H, Sun Z, Li SH, Wu J, Fazel S, Weisel RD, Rakowski H, Lindner J and Li RK: Ultrasound-targeted gene delivery induces angiogenesis after a myocardial infarction in mice. JACC Cardiovasc Imaging 2: 869-879, 2009.

12. Castle J, Butts M, Healey A, Kent K, Marino M and Feinstein SB: Ultrasound-mediated targeted drug delivery: Recent success and remaining challenges. Am J Physiol Heart Circ Physiol 304: H350-H357, 2013.

13. Delalande A, Postema M, Mignet N, Midoux P and Pichon C: Ultrasound and microbubble-assisted gene delivery: Recent advances and ongoing challenges. Ther Deliv 3: 1199-1215, 2012.

14. Yang $\mathrm{H}, \mathrm{Xiong} \mathrm{XY}$, Zhang L, Wu C and Liu Y: Adhesion of bio-functionalized ultrasound microbubbles to endothelial cells by targeting to vascular cell adhesion molecule-1 under shear flow. Int J Nanomedicine 6: 2043-2051, 2011.

15. Murciano JC, Muro S, Koniaris L, Christofidou-Solomidou M, Harshaw DW, Albelda SM, Granger DN, Cines DB and Muzykantov VR: ICAM-directed vascular immunotargeting of antithrombotic agents to the endothelial luminal surface. Blood 101: 3977-3984, 2003.

16. Yan Y, Liao Y, Yang L, Wu J, Du J, Xuan W, Ji L, Huang Q, Liu Y and Bin J: Late-phase detection of recent myocardial ischaemia using ultrasound molecular imaging targeted to intercellular adhesion molecule-1. Cardiovasc Res 89: 175-183, 2011.

17. Morisada T, Kubota Y,Urano T, Suda T and Oike Y: Angiopoietins and angiopoietin-like proteins in angiogenesis. Endothelium 13: 71-79, 2006.

18. Zhang H, Yuan YL, Wang Z, Jiang B, Zhang CS, Wang Q, Xu XH, Dong HY and Zhang ZM: Sequential, timely and controlled expression of hVEGF165 and Ang-1 effectively improves functional angiogenesis and cardiac function in vivo. Gene Ther 20: 893-900, 2013.

19. Paul A, Binsalamah ZM, Khan AA, Abbasia S, Elias CB Shum-Tim D and Prakash S: A nanobiohybrid complex of recombinant baculovirus and Tat/DNA nanoparticles for delivery of Ang-1 transgene in myocardial infarction therapy. Biomaterials 32: 8304-8318, 2011.

20. Fagiani E and Christofori G: Angiopoietins in angiogenesis. Cancer Lett 328: 18-26, 2013.

21. Xie A, Belcik T, Qi Y, Morgan TK, Champaneri SA, Taylor S Davidson BP, Zhao Y, Klibanov AL, Kuliszewski MA, et al: Ultrasound-mediated vascular gene transfection by cavitation of endothelial-targeted cationic microbubbles. JACC Cardiovasc Imaging 5: 1253-1262, 2012.

22. Yan P, Chen KJ, Wu J, Sun L, Sung HW, Weisel RD and Li RK: The use of MMP2 antibody-conjugated cationic microbubble to target the ischemic myocardium, enhance Timp3 gene transfection and improve cardiac function. Biomaterials 35: 1063-873, 2014.

23. Weidner N: Current pathologic methods for measuring intratumoral microvessel density within breast carcinoma and other solid tumors. Breast Cancer Res Treat 36: 169-180, 1995.

24. Delalande A, Kotopoulis S, Postema M, Midoux P and Pichon C: Sonoporation: Mechanistic insights and ongoing challenges for gene transfer. Gene 525: 191-199, 2013.

25. Panje CM, Wang DS, Pysz MA, Paulmurugan R, Ren Y, Tranquart F, Tian L and Willmann JK: Ultrasound-mediated gene delivery with cationic versus neutral microbubbles: Effect of DNA and microbubble dose on in vivo transfection efficiency. Theranostics 2: 1078-1091, 2012.

26. Luo D and Saltzman WM: Enhancement of transfection by physical concentration of DNA at the cell surface. Nat Biotechnol 18: 893-895, 2000 
27. Wang DS, Panje C, Pysz MA, Paulmurugan R, Rosenberg J, Gambhir SS, Schneider M and Willmann JK: Cationic versus neutral microbubbles for ultrasound-mediated gene delivery in cancer. Radiology 264: 721-732, 2012.

28. Wang X, Liang HD, Dong B, Lu QL and Blomley MJ: Gene transfer with microbubble ultrasound and plasmid DNA into skeletal muscle of mice: Comparison between commercially available microbubble contrast agents. Radiology 237: 224-229, 2005.

29. Zhou J, Wang Y, Xiong Y, Wang H, Feng Y and Chen J: Delivery of TFPI-2 using ultrasound with a microbubble agent (SonoVue) inhibits intimal hyperplasia after balloon injury in a rabbit carotid artery model. Ultrasound Med Biol 36: 1876-1883, 2010.

30. Lentacker I, De Geest BG, Vandenbroucke RE, Peeters L, Demeester J, De Smedt SC and Sanders NN: Ultrasound-responsive polymer-coated microbubbles that bind and protect DNA. Langmuir 22: 7273-7278, 2006.

31. Ju J, Huan ML, Wan N, Hou YL, Ma XX, Jia YY, Li C, Zhou SY and Zhang BL: Cholesterol derived cationic lipids as potential non-viral gene delivery vectors and their serum compatibility. Bioorg Med Chem Lett 26: 2401-2407, 2016.

32. Sun L, Huang CW, Wu J, Chen KJ, Li SH, Weisel RD, Rakowski H, Sung HW and Li RK: The use of cationic microbubbles to improve ultrasound-targeted gene delivery to the ischemic myocardium. Biomaterials 34: 2107-2116, 2013.
33. Wang X, Hagemeyer CE, Hohmann JD, Leitner E, Armstrong PC, Jia F, Olschewski M, Needles A, Peter K and Ahrens I: Novel single-chain antibody-targeted microbubbles for molecular ultrasound imaging of thrombosis: Validation of a unique noninvasive method for rapid and sensitive detection of thrombi and monitoring of success or failure of thrombolysis in mice. Circulation 125: 3117-3126, 2012.

34. Warram JM, Sorace AG, Mahoney M, Samuel S, Harbin B, Joshi M, Martin A, Whitworth L, Hoyt K and Zinn KR: Biodistribution of P-selectin targeted microbubbles. J Drug Target 22: 387-394, 2014.

35. Leong-Poi H, Christiansen J, Klibanov AL, Kaul S and Lindner JR: Noninvasive assessment of angiogenesis by ultrasound and microbubbles targeted to alpha (v)-integrinss. Circulation 107: 455-460, 2003.

36. Dabek J, Ligus J and Szota J: Oligonucleotide microarray and QRT-PCR study of adhesion protein gene expression in acute coronary syndrome patients. Inflammation 33: 398-407, 2010.

37. Lawson $C$ and Wolf S: ICAM-1 signaling in endothelial cells. Pharmacol Rep 61: 22-32, 2009.

38. Mulvihill NT and Foley JB: Inflammation in acute coronary syndromes. Heart 87: 201-204, 2002.

39. Benson V, McMahon AC and Lowe HC: ICAM-1 in acute myocardial infarction: A potential therapeutic target. Curr Mol Med 7: 219-227, 2007 